\title{
Whole-body motion of a Humanoid robot for passing through a door - Opening a door by impulsive force -
}

\author{
Hitoshi Arisumi, Jean-Rémy Chardonnet, and Kazuhito Yokoi, Member, IEEE
}

\begin{abstract}
There are many kinds of large, heavy objects, or objects with geometrical constraints in our daily life, but non-fixed robots such as humanoid robots are still not able to manipulate them sufficiently well. In this paper we focus on a swing door as a heavy object with geometrical constraints, and present a method for the humanoid robots to open it by using impulsive forces. We first discuss on momentum transfer from the robot to the door. Then we propose a method of generating a whole body motion to impact on the door. We analyze the dynamic model of the door, and we confirm the validity of our method through simulation. At last, we realize a motion of the robot opening a swing door quickly by the method in experiment with the HRP-2 robot hardware.
\end{abstract}

Index Terms - Humanoid robot, Dynamic whole body manipulation, ZMP, Impact

\section{INTRODUCTION}

$\mathrm{I}_{\mathrm{v}}^{\mathrm{N}}$ $\mathrm{N}$ the near future we expect humanoid robots to perform various tasks in offices, hospitals or homes for elderly people, more generally in our living environment. In order to realize our daily tasks such as objects manipulation or guidance as illustrated in Fig. 1, robots must be able to move to many different places. However various obstacles can prevent them from moving freely, for instance doors are troublesome obstacles which robots cannot easily pass through. Among many kinds of doors, swing doors used in office are more difficult to manipulate because of restoring forces and its weight. We address this matter for the first step so that non-fixed robots such as humanoid robots can manipulate heavy objects or objects with geometrical constraints.

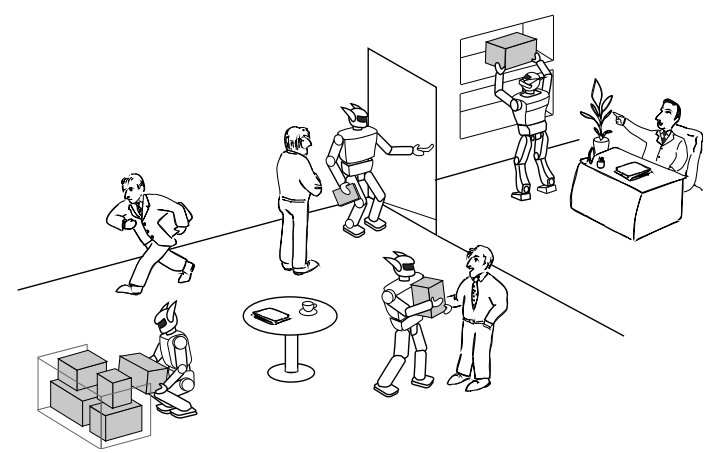

Fig. 1. Robotic porter and guider system

Manuscript received March $1^{\text {st }}$, 2009. H. Arisumi (Corresponding Author), J-R Chardonnet, and K. Yokoi are with the Intelligent Systems Research Institute of the National Institute of Advanced Industrial Science and Technology (AIST), Tsukuba Central 2, 1-1-1 Umezono, Tsukuba, Ibaraki 305-8568, Japan. Contact TEL: +81-29-861-7282 FAX: +81-29-861-5444 e-mail: h-arisumi@aist.go.jp
In previous works, several methods of opening a door statically by wheeled robots are proposed [1]-[3]. But these works do not use a robot's motion approaching to a door to open it. Besides they do not discuss about stability of the robots. As for the pushing manipulation by humanoid robots [4], [5], a door is not a target object. They proposed the methods of pushing an object such as a box or a wheelchair statically by continuous forces considering the displacement of the center of mass of the robot. These methods may be efficient to open a light door with small reaction forces such as a joint friction. On the other hand, in case of heavy doors, doors shut tight, or doors with restoring forces such as swing doors, the biggest power is necessary at the beginning of opening them. Therefore robots may not open them statically because a static motion depends on not a motion of robot's links but just a continuous power of actuators. To solve this problem we propose here to apply the impact manipulation to open them dynamically.

Impact manipulation has been used in several works. For example in [6], a manipulator hits a disc to the desired position on a slippery floor like in ice-hockey, which is especially called release manipulation. As the manipulator used in this work has a fixed base, stability is out of concern. Matsumoto et al. [7] succeeded in making a humanoid robot break a wooden board by applying impulsive forces. However compared to the weight of the robot, the board is very light. Moreover manipulation of the object after the impact is not discussed. In our earlier works, we succeeded in making a humanoid robot lift an object by using a preliminary motion and applying impulsive forces without moving its feet [8].

In this paper, a motion of a humanoid robot getting closer to a door is regarded as a preliminary motion for generating its momentum. We then discuss on a method of opening a swing door at a desired angle by impact where the momentum is transferred from the robot to a door. The paper is organized as follows. We first discuss on the way for humanoid robots to approach to a door, to open it, and to support it. We also analyze the structure of a swing door and formulate its dynamic model. Furthermore we deal with the momentum transfer from the robot to the door then we propose a motion planning to apply an impact on the door. Simulation of the motion for opening the door at a desired angle is then performed in Section III. In Section IV, we identify the dynamic parameters of the swing door, and we confirm the availability of the proposed method through experiments. We conclude with a summary in the final section. 


\section{PRELIMINARY MOTION FOR MANIPULATING A DOOR}

\section{A. Strategy for opening a door}

When the robot hits a door, a large impulsive force to the door can be generated. Then an impact manipulation is available for a swing door because large force is needed at the beginning of opening it. The first question we should ask is how the humanoid robot hits the door. It is possible to use arms or feet to punch or kick the door, but it may not generate enough momentum, or it can cause the instability of the robot. On top of that, motion of arms is restricted when carrying an object. Against these matters, we consider that the humanoid robot hits the door with its whole body which is regarded as an integrated mass of robot's links.

As for the first step, we address the case that the robot hits the door with its upper body which constitutes a large proportion of the robot's mass. There are mainly three ways for the humanoid robot to hit the door: at the front, at the back, or at the side. In case of hitting at the front, the knee is apt to interfere with the door, and that may cause a tumble of the robot. On the other hand, hitting at the back is a possible way but the workspace of the arm is then restricted, thus making difficult the manipulation of the door after the impact. Consequently, we discuss on a method to apply impulsive forces to the door by hitting it at the side, as illustrated in Fig. 2. When the robot walks, the ZMP of the robot moves one support polygon of foot to another. Thus in general, it makes the upper body of the robot oscillate right and left. We use here this oscillation from side to side for the preliminary motion to generate the momentum. In this paper, we focus on the way to displace the center of mass derived by one lateral step to the door.

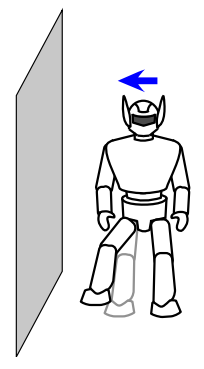

(a) stepping forth

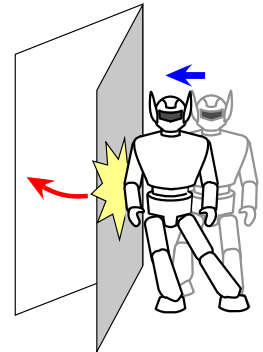

(b) hitting a door

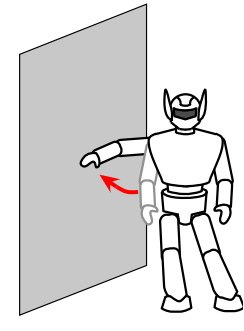

(c) supporting a door
Fig. 2. Target motion

When the door stops opening, the robot extends its arm to prevent the door from shutting again as shown in Fig. 2 (c). It is the easiest way to stop a motion of the door after the impact. In addition, it makes good use of the kinetic energy of the door for opening it. Moreover, the robot can support the door stably because the door is opened to an angular range where the restoring force is enough small.

\section{B. Model of the robot and the door}

The model of both the robot and the door is described in Fig. 3. We set the origin of the reference frame at the middle between the feet at the initial configuration, the $y$ axis in the opposite direction of pushing, and the $z$ axis upward. In the figure, $m_{r o b}$ denotes the robot's mass, $I_{r o b}$ denotes the inertia moment around the line of the $x$-direction passing through the center of mass (CoM) of the robot. Furthermore, $g$ denotes the gravitational acceleration. We also represent the model of the door from the top view in the right part of Fig. 3. Point A represents the door's hinge, point B is the point where the robot impacts, $I_{A}$ is the angular momentum of the door around its hinge (namely point $\mathrm{A}$ ), $l_{B}$ is the distance between points $\mathrm{A}$ and $\mathrm{B}, \theta$ is the opening angle, $\omega$ ' is the initial angular velocity of the door when it opens, and $a_{r}$ is the height between the CoM of the robot and impact point $\mathrm{B}$.

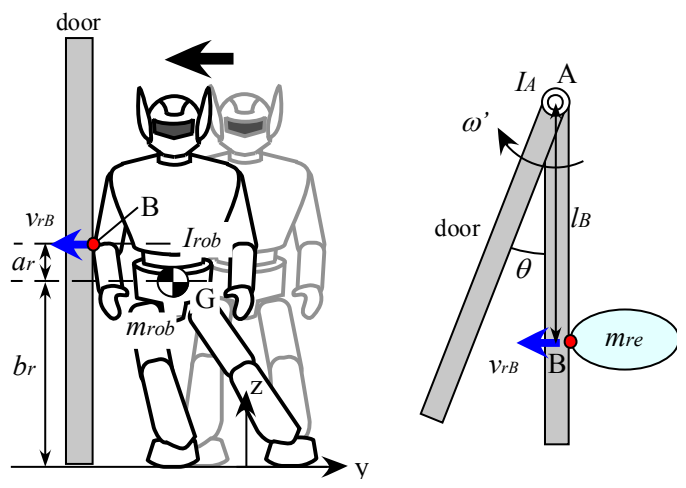

Fig.3. Model of the robot and a door

The door closer which is equipped on the top of the swing door controls a behavior of the swing door. We explain here its mechanism.

The door closer consists in a four-link mechanism as shown in Fig. 4. This system can be modeled as in the right part of Fig. 4.
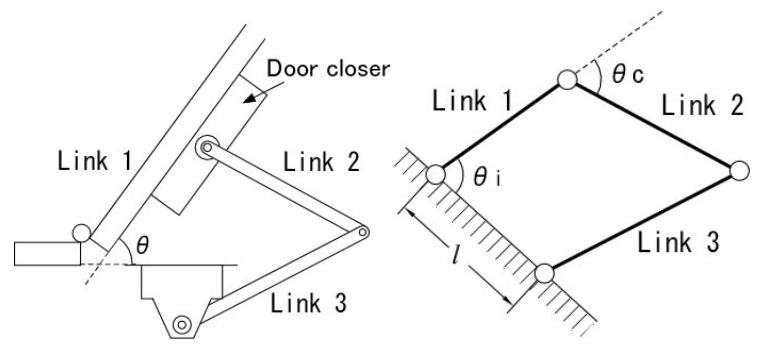

Fig.4. Door closer modeled by a four-link mechanism

Modeling the joint of the door closer as a spring-damper system, the dynamic equation of the door is given by

$$
\tau_{\text {ext }}=I_{A} \ddot{\theta}+c_{c}\{\eta(\theta)\}^{2} \dot{\theta}+\eta(\theta)\left\{k_{c} f(\theta)+\tau_{c 0}^{\prime}\right\},
$$

where $\tau_{\text {ext }}$ denotes the external torque that applies to the door around the hinge rod, $c_{c}$ is the viscosity coefficient of the door closer, $\eta(\theta)$ is corresponding to a reduction gear ratio, $k_{c}$ is the torsional spring coefficient, $f(\theta)$ is the angle of the door closer, and $\tau^{\prime}{ }_{c 0}$ is the initial torque. The identification of $\eta(\theta)$ and $f(\theta)$ is mentioned in the Appendix at the end of this paper. Note that the motion of the door with the spring-damper model set at the joint of the door closer is different from that with the same model set at the joint of the door itself. As shown in Fig. 
16 in Appendix, the restoring force which depends on the spring of the door closer is the biggest at the beginning of opening the door.

In the next paragraph, we discuss on the motion generation of the robot taking into account the following constraints:

1) The robot impacts the door in a fixed configuration of its upper part.

2) The robot impacts in the negative direction of $y$ axis (meaning the motion of the CoM is along the $y$ axis)

3) Target object is the swing door with a door closer whose model is described above.

4) When the door starts to open, the robot does not contact it until the door has opened to its maximum angle. Then it extends its arm to maintain the door open.

\section{Motion generation}

Here we decide the motion of each step in reverse order, meaning from the end state to the initial state. door

\section{1) Standing position of the robot and opening angle of the}

First, as in Fig. 2(a), we decide the position of the foot that steps forth, so that it enables the robot to impact the door. As shown in Fig. 5(a), we fix the arm close to the upper body of the robot. As the configuration of the upper body is fixed, the $y$-coordinate of the CoM at the impact can be decided by letting the arm touch the door as shown in Fig. 5(a). In order to walk ahead smoothly after the impact, it is desirable to move the rear foot (here the left foot) to the front (toward the door's side). This means that the GCoM (Grand projection of the Center of Mass) must remain inside the support polygon of the right foot when impacting. Here we can decide the position of the right foot by setting the GCoM at the inner edge of the support polygon of the right foot, as point $\mathrm{C}$ shown in Fig. 5(a). To decide the position of the robot along the $x$ axis represented in Fig. 5(b), we need to consider the geometrical and dynamical constraints such that the robot can reach and stably hold the door which is opened to the desired angle.

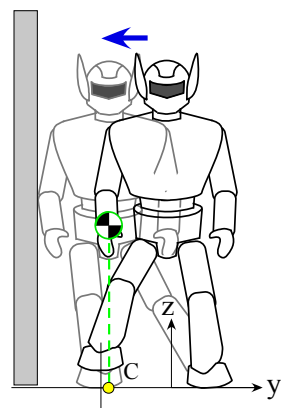

(a) side view

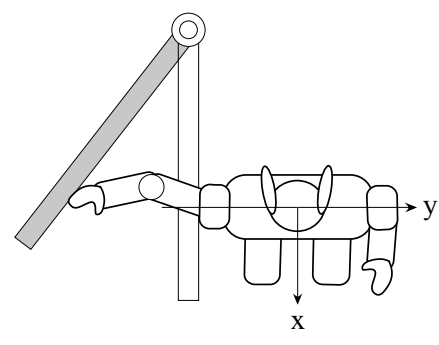

(b) upper view
Fig.5. Standing position of the robot and opening angle of a door

As shown in Fig. 16 in Appendix, the more the door opens, the more the restoring torque decreases. To reduce the restoring force, the door should be open wider, and the robot should hold it at the contact point further from its hinge. We decide the $x$-coordinate of the robot at the impact so that the position of the arm is the furthest from the hinge of the door with considering the arm total length and the maximum allowable restoring force. The maximum allowable restoring force is set as the disturbance which can be compensated by the stabilizer of the robot. The desired opening angle of the door can be derived by the position of the robot at the impact.

\section{2) Desired initial angular velocity of the door}

We describe here a method to get the initial angular velocity of the door that is needed to open to the desired angle. Giving the initial angle and the final opening angle of the door, we obtain the initial angular velocity by the numerical approach using (1). We neglect the motion of the door during the impact because the robot contacts the door in a very short time. Just after the impact, we set $\tau_{e x t}$ at zero because the door does not receive any external forces.

\section{3) Momentum transfer}

During the impact, the relation between the momentum of the door and the robot can be expressed by using the law of conservation of angular momentum around the point A in Fig. 3 as follows:

$$
m_{r e} v_{r B} l_{B}=m_{r e} v_{r B}^{\prime} l_{B}+I_{A} \omega^{\prime}
$$

where $m_{r e}$ is the effective mass of the robot at impact point B, $v_{r B}$ and $v_{r B}^{\prime}$ are the pre-impact and post-impact velocities of the robot respectively. The effective mass of the robot $m_{r e}$ can be approximately expressed by

$$
m_{r e}=\frac{I_{r o b} m_{r o b}}{I_{r o b}+a_{r}^{2} m_{r o b}} .
$$

From (2) and (3), we get the velocity of impact point B when the robot impacts the door as follows:

$$
v_{r B}=\frac{\left(I_{r o b}+a_{r}^{2} m_{r o b}\right) I_{A}}{I_{r o b} m_{r o b} l_{B}} \omega_{B}^{\prime}+v_{r B}^{\prime} .
$$

4) Configuration of the robot and motion of the center of mass

First we decide the configuration of the robot when impacting. In order to avoid any slip of the foot during the impact, we locate the center of percussion at point $\mathrm{C}$ in Fig. 5. Then the relation between the height of the CoM of the robot $b_{r}$ and the height of the impact point $a_{r}$ in Fig. 3 is as follows:

$$
b_{r}=I_{r o b} /\left(m_{r o b} a_{r}\right) .
$$

When giving the height of the upper body and positions of both feet at the impact, the configuration of the robot is uniquely decided. Thus $I_{r o b}$ and $b_{r}$ are computed according to the configuration of the robot. On the other hand, $a_{r}$ depends on the position of the CoM and the contact point. In this paper we assume that the contact point is located at the elbow of the 
robot. Hence $a_{r}, b_{r}$, and $I_{r o b}$ which satisfy (5) are obtained by changing the height of the upper body.

In the next step, we design the motion of the robot before the impact. To simplify the problem, we fix the configuration of the upper body and the height of the hip while the robot moves to the door. When the upper body is fixed, the velocity of the robot at point B $v_{r B}$ is equal to the velocity of the CoM of the robot $v_{\text {rob }}$. As mentioned in the previous paragraph, the needed initial angular velocity of the door $\omega$ ' is given by the desired opening angle. Furthermore as mentioned earlier, the velocity of the robot just before the impact $v_{r B}$ is given by (4) when setting the configuration at the impact and the velocity of the robot just after the impact $v_{r B}^{\prime}$. There are many ways to generate this velocity $v_{r B}$. For instance, the cam curve is a suitable way to accelerate the robot smoothly. We use here the modified constant velocity cam curve [9] for the trajectory of the CoM of the robot. It represents in Fig. 6 with normalized axes. The feature of this curve is that the velocity remains constant for a large period as shown in the figure. Taking into account the time delay of impact, the velocity of the robot before impact should be constant. In this paper, we use the first half period of the curves represented within the blue dotted lines in Fig. 6, and consider that the impact occurs at the half time of the curve. We thus design the trajectory for the CoM of the robot during this period. We skip the detailed description for the design of this trajectory due to limitations of space.

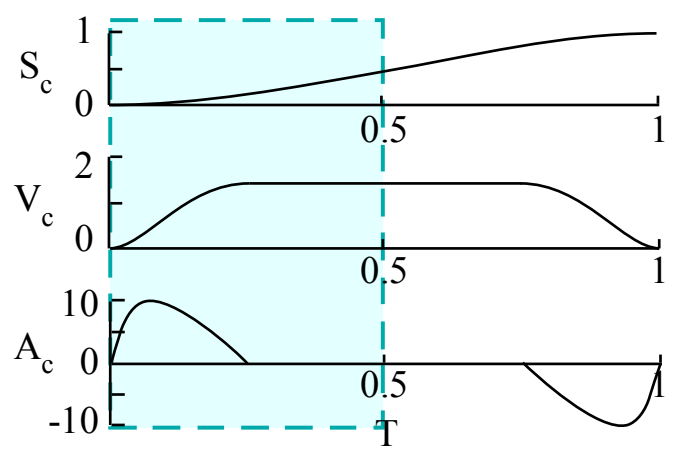

Fig.6. Modified constant velocity cam curve

When the door stops opening after the impact, the restoring torque is given by (12) in Appendix. Then we can calculate the external force from the door to the robot when the robot holds the door. Hence the GCoM of the robot at that time is calculated so that the robot keeps its balance against the external force.

\section{Simulation}

We conduct a simulation on the method presented in the previous section using the OpenHRP3 [10], a dynamic simulator, to open a swing door with a door closer that generates restoring forces. Snapshots of the simulation are presented in Fig. 7. We have the following values: $m_{r o b}=58 \mathrm{~kg}$, $I_{r o b}=6.147 \mathrm{kgm}^{2}, b_{r}=0.71 \mathrm{~m}$, and $l_{B}=0.42 \mathrm{~m}$. The mass of the door is $47.62 \mathrm{~kg}$ which is more than $82 \%$ of that of the robot.
We set the maximum desired opening angle at $25 \mathrm{deg}$. The dynamics parameters of the door are as follows: $I_{A}=$ $12.75 \mathrm{kgm}^{2}, c_{c}=0.06 \mathrm{Ns}$, and $k_{c}=1.13 \mathrm{Nm} / \mathrm{rad}$.

In Fig. 7, the center of mass of the robot moves from the initial state in (a) to the state in (b), i.e. to be inside the left foot. Then the right foot approaches the door as shown in (c). In (d), the upper body moves horizontally and impacts the door. The kinetic momentum of the robot decreases at that time and is transferred to the door, hence the door opens as shown in (e). In (f), the robot extends its hand to hold the door and prepares for the next walk step.
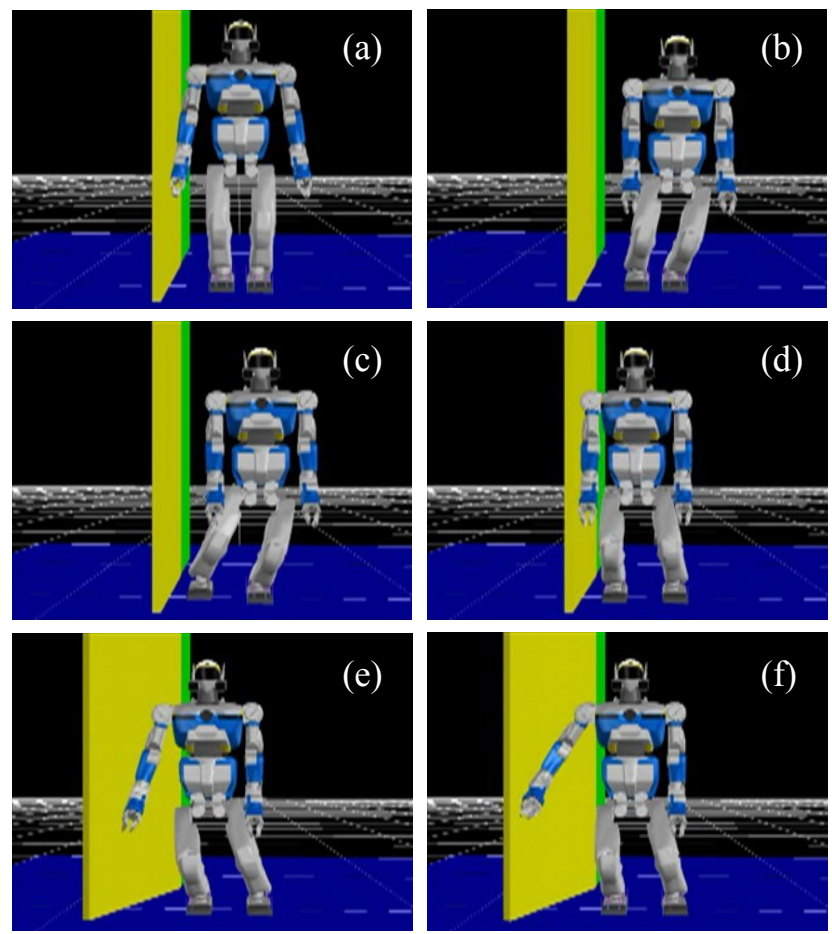

Fig. 7. Snapshot of the motion of HRP-2 opening a door

In Fig. 8, we compute the position of the ZMP using the measurement value of the force sensors at the feet. The upper (blue) and lower (green) broken lines are the position of the left and right ankles along the $y$ axis, respectively. The grey zone in the figure represents the support polygon in the $y$-direction. The concave part between $8.6 \mathrm{~s}$ and $10.3 \mathrm{~s}$ is the single support zone, while the other parts represent that the robot is in double support. The brown vertical broken line represents the instant of impact. After the impact, the ZMP oscillates because the robot's hand collides with the door to support it. As shown in this figure, the ZMP remains within the limits of the support polygon, meaning the generated motion to open the door is stable.

Fig. 9 shows the opening angle of the door when the robot gives an impact on it. The red dotted line represents the desired opening angle. As shown in the figure, the robot touches the door after it starts closing from the maximum opening angle, and pushes it back to the desired angle. At that time, the robot receives an impact from the door, but its effect can be regarded as small judging from the variation in the 
ZMP shown in Fig. 8. The measured opening angle exceeds the desired maximum angle by its $5 \%$, but this may due to the effects of the stabilizer on the motion during the impact.

In simulation, we showed the availability of our proposed method of generating the robot's motion to open a door to a desired angle by exerting impulsive forces and holding it.

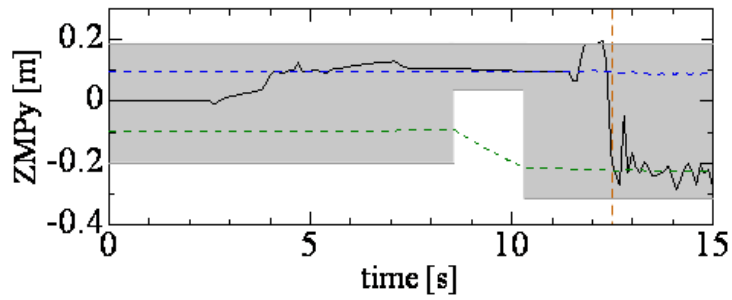

Fig. 8. ZMP in the negative direction of $y$

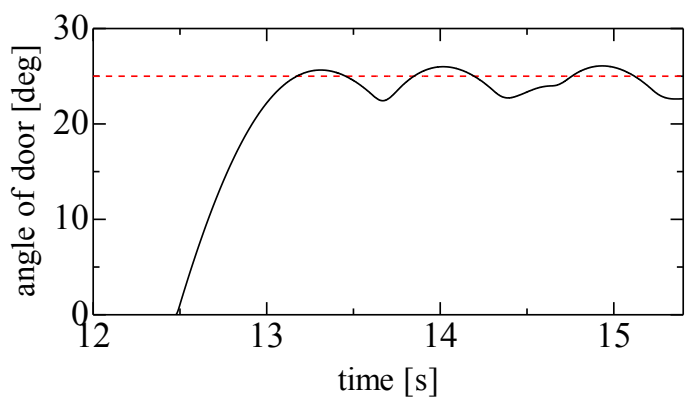

Fig. 9. Door motion after impact

\section{EXPERIMENT}

\section{A. Identification of the dynamic model of the swing door}

In order to identify the dynamic model of the swing door, we conduct basic experiments. A mechanical attachment with a measurement device is set at the joint of a door, as shown in Fig. 10. For the measurement device we use the encoder MES-20-3600P (Microtech Lab. Inc.) whose resolution is 3600 pulse/rev. We achieve the high accuracy by multiplying the number of input pulses on the counterboard by four. This system runs on a $1 \mathrm{GHz}$ CPU-based PC (Intel Pentium ${ }^{(\mathrm{R})}$ III) under ART-Linux 2.4.34. The sampling time is set at $0.001 \mathrm{sec}$.

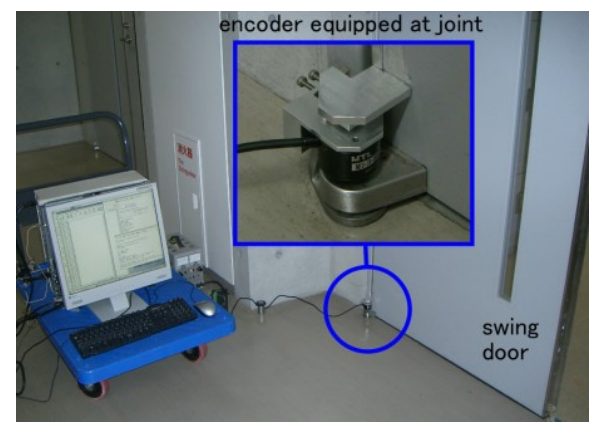

Fig. 10. Device for measuring the angle of door

We give several impacts on the door by hand and measure the angle of the door. Impacts are made within a range of opening angles that are smaller than 10 degrees. The black solid curves in Fig. 11 are the angles of the door from 10 degrees to the maximum opening angle when giving impacts We then make (1) fit these data as much as possible by changing parameters that appear in (1) to identify them. Concretely speaking, we change parameters, $I_{A}, c_{c}, k_{c}$, and $\tau^{\prime}{ }_{c 0}$ within each limit to minimize the following function $E_{f i t}$ :

$$
E_{f i t}=\sum_{i=0}^{i_{e}}\left(\theta_{\text {exp }}\left(t_{i}\right)-\theta_{\text {sim }}\left(t_{i}\right)\right)^{2}
$$

where $i$ is a time step, $\theta_{\exp }\left(t_{i}\right)$ and $\theta_{\text {sim }}\left(t_{i}\right)$ are the experimental and simulation data of angle of the door, respectively. $\theta_{\text {sim }}\left(t_{i}\right)$ is calculated by (1) numerically when giving the parameters.

We finally obtain the identified values here correspond to those mentioned in the previous section. The theoretical values computed by identification are represented in broken line in Fig. 11. We can see that the theoretical values match well experimental data.

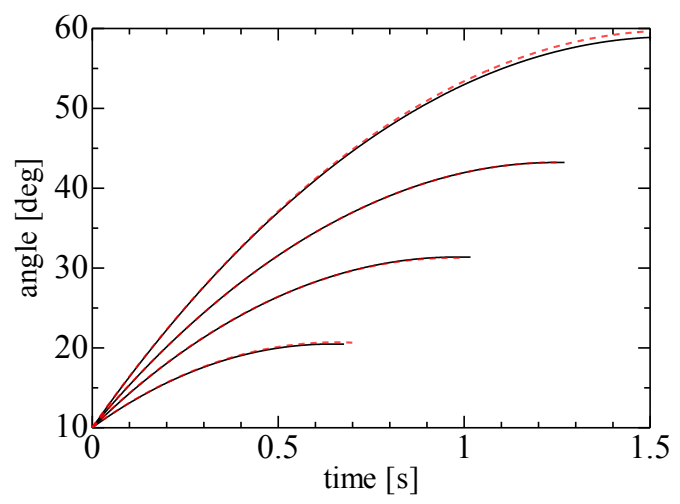

Fig. 11. Dynamic behavior of a swing door when giving several impacts on it

\section{B. Door opening by HRP-2}

We perform experiments of the door opening motion by using the humanoid robot HRP-2 developed by Kawada industries Inc. The number of d.o.f. of HRP-2 is 30. The height and the weight of the HRP-2 are $h=1.539 \mathrm{~m}$ and $m_{r o b}=$ $58 \mathrm{~kg}$, respectively.

For safety reasons, we skip the robot's motion to hold the door in the experiment. Fig. 12 shows the experimental results with HRP-2 impacting a swing door. Each motion except (e) and (f) in Fig. 12 is quite similar to the motion shown in Fig. 7. In Fig. 12 (f), the robot comes back in the room to avoid the impact of the closing door.

As shown in Fig. 13, the ZMP curve changes within the grey area which is the same as Fig. 8. Compared to simulation results, the ZMP moves in a more stable way but this may due to the damping effect of the foot's sole and the floor. Fig. 14 shows the opening angle of the door after the impact with the time. Because of the damping effect of the door closer, opening and closing are not symmetric. However, in this paper we do not consider closing but opening. We can observe that the opening angle reaches the desired angle with an error of not over $2 \mathrm{deg}$.

We proved the practicability of the proposed method from the viewpoint of stability. 

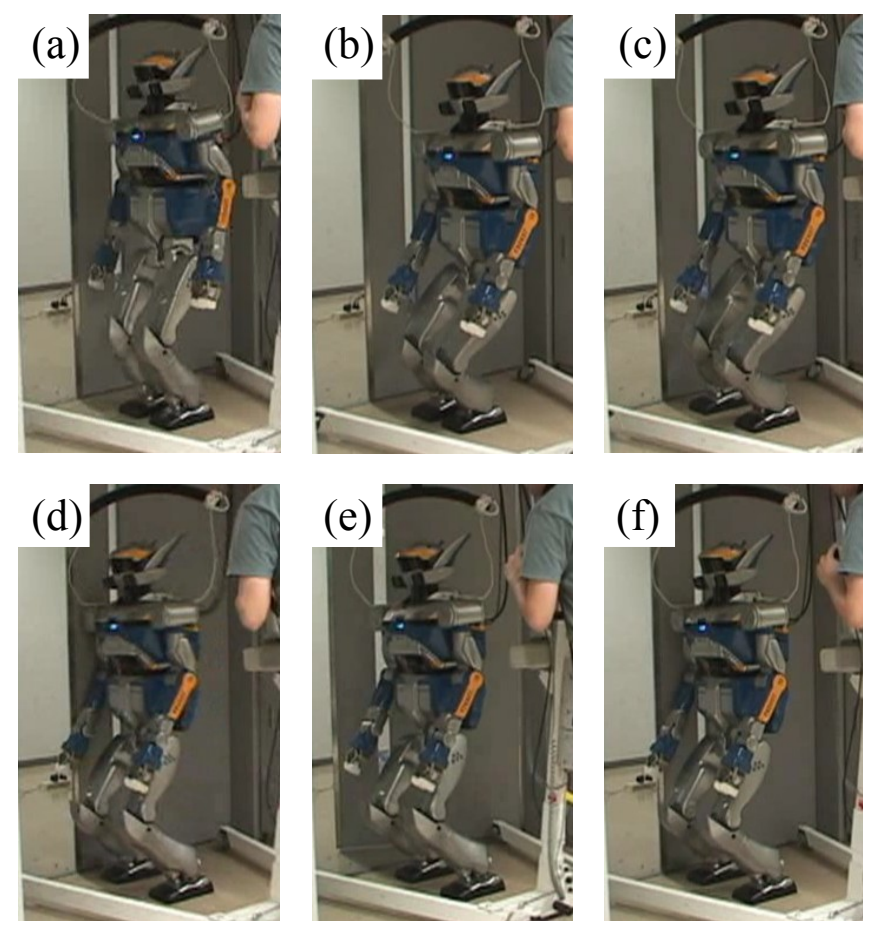

Fig. 12. Impact manipulation for opening a swing door

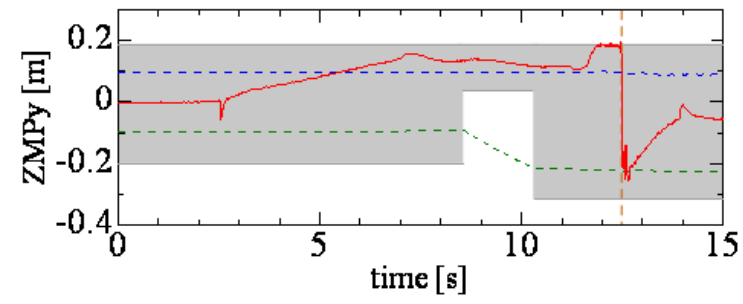

Fig.13. ZMP of the robot (experimental result)

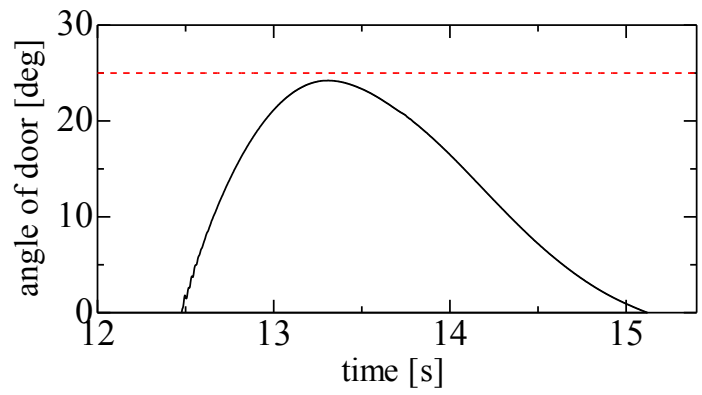

Fig. 14. Door motion after impact (experimental result)

\section{CONCLUSION}

In this paper, we addressed a manipulation of a heavy object with a geometrical constraint by the humanoid robot. Focusing on a swing door which we need a large force to open, we proposed the method of manipulating the door by impact which is generated by a whole-body motion of the robot. We firstly identified the dynamic model of the swing door through basic experiments. We then proposed a motion generation for the robot, and we demonstrated the availability of our method through a dynamic simulation. We finally conducted experiments on a humanoid robot, and realized our stable and fast motion of the robot opening the swing door whose mass is more than $80 \%$ of that of the robot.

Future works are to develop a parallel control of manipulation and locomotion for a smooth motion of the humanoid robot passing through the door, and to realize it with the robot hardware.

\section{APPENDIX}

\section{A. Analysis of the structure of the swing door with door closer}

We give here more detailed description of the mechanism of the door closer. Especially we explain the adjusting function of controlling the motion of the door by taking the four-link model of Fig. 4.

In Fig. 4, $\theta$ denotes the opening angle of the door, and we set it at $0 \mathrm{rad}$ when the door is closed. $\theta_{i}$ is the angle between the fixed part and link $1, \theta_{c}$ is the angle between link 1 and link 2 , and $l$ is the distance between the fixed points of link 1 and link 3. $\theta$ is expressed by

$$
\theta_{i}=\theta+\theta_{i 0}
$$

where $\theta_{i 0}$ is an initial value of $\theta_{i}$, when $\theta$ is equal to zero. Letting the lengths of links 1,2 and 3 as $l_{1}, l_{2}$, and $l_{3}$, respectively, $\theta_{c}$ is given by the following equation

$$
\begin{aligned}
\theta_{c}= & f(\theta)=\pi-\cos ^{-1} \frac{l_{1}-l \cos \left(\theta+\theta_{i 0}\right)}{\sqrt{l^{2}+l_{1}^{2}-2 l_{1} l \cos \left(\theta+\theta_{i 0}\right)}} \\
& -\cos ^{-1} \frac{l^{2}+l_{1}^{2}+l_{2}^{2}-l_{3}^{2}-2 l_{1} l \cos \left(\theta+\theta_{i 0}\right)}{2 l_{2} \sqrt{l^{2}+l_{1}^{2}-2 l_{1} l \cos \left(\theta+\theta_{i 0}\right)}} .
\end{aligned}
$$

The stiffness and the viscosity of the damper are directly linked to the angular position $\theta_{c}$ and the angular velocity $\dot{\theta}_{c}$. Differentiating (8) with respect to $\theta$, we obtain

$$
\eta(\theta)=\frac{d \theta_{c}}{d \theta}
$$

where $\eta(\theta)$ corresponds to a damping coefficient. According to the principle of virtual work, the relationship between the torque exerted on the door $\tau$ and the torque exerted on the closer $\tau_{c}$ is expressed by $\tau d \theta=\tau_{c} d \theta_{c}$. Then we get

$$
\tau=\eta(\theta) \tau_{c}
$$

If we consider that the torsional spring's stiffness coefficient $k_{c}$ is constant, we can express the restoring force $\tau_{c 1}$ as follows:

$$
\tau_{c 1}=k_{c}\left(\theta_{c}-\theta_{c 0}\right)+\tau_{c 0}
$$

where $\theta_{c 0}=f(0)$, and $\tau_{c 0}$ is the torque of the door closer when $\theta_{c}$ $=\theta_{c 0}$. Substituting (8) and (11) into (10), we get

$$
\tau_{1}=\eta(\theta)\left\{k_{c} f(\theta)+\tau_{c 0}^{\prime}\right\},
$$


where $\tau_{c 0}^{\prime}=\tau_{c 0}-k_{c} \theta_{c 0}$.

Letting the viscosity coefficient of the spring as $c_{c}$, the damping torque $\tau_{c 2}$ is given by $\tau_{c 2}=c_{c} \dot{\theta}_{c}=c_{c} \eta(\theta) \dot{\theta}$ from (9). Substituting $\tau_{c 2}$ into (10), we obtain

$$
\tau_{2}=c_{c}\{\eta(\theta)\}^{2} \dot{\theta}
$$

By combining $\tau_{1}$ and $\tau_{2}$ with the inertial torque of the door, we obtain the dynamic equation of the door (1).

\section{B. Analysis of the motion of the swing door}

Giving $l=195 \mathrm{~mm}, l_{1}=204 \mathrm{~mm}, l_{2}=220 \mathrm{~mm}, l_{3}=265 \mathrm{~mm}$, and $\theta_{i 0}=0.34 \mathrm{rad}$, the relation between the angle of the door closer $\theta_{c}$ and the angle of the door $\theta$ is shown in Fig. 15.

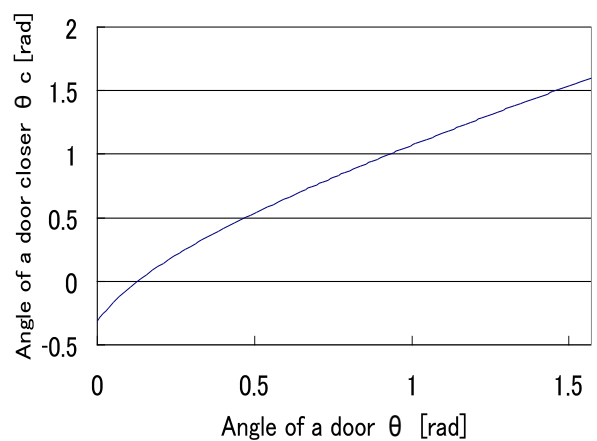

Fig. 15 Relationship between angles of the door and the door closer

We also show the restoring force of the door that was measured using digital force gauge in Fig. 16 [11]. As shown in this graph, the restoring force gets the maximum value when the door starts to open, and decreases smoothly as the door opens further. From these results, we set the parameters of the spring of the door using (12). Our modeled torque is then represented in pink in Fig. 16.

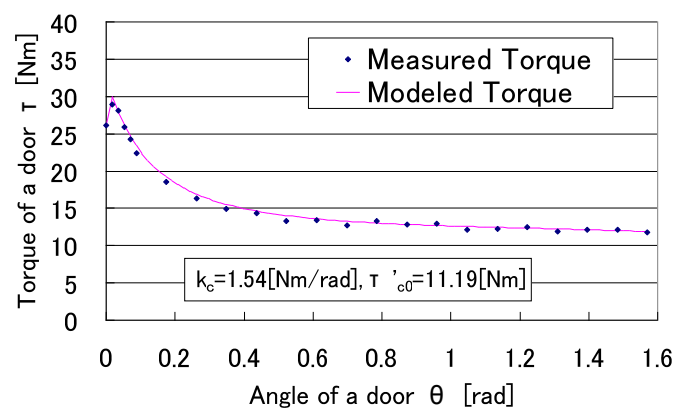

Fig. 16 Restoring torque with respect to angle of the door

\section{REFERENCES}

[1] L. Petersson, D. Austine, and D. Kragic, "High-level control of a mobile manipulator for door opening," Proc. of the IEEE/RSJ International Conference on Intelligent Robots and Systems (IROS'00), 2000.

[2] C. Rhee, W. Chung, M. Kim, Y. Shim, and H. Lee, "Door opening control using the multi-fingered robotic hand for the indoor service robot," Proc. of the IEEE Int. Conf. on Robotics and Automation (ICRA'04), 2004.

[3] Anna Petrovskaya and Andrew Y. Ng, "Probabilistic Mobile Manipulation in Dynamic Environments, with Application to Opening Doors," International Joint Conferences on Artificial Intelligence (IJCAI), pp. 2178-2184, 2007.

[4] K. Harada, S. Kajita, K. Kaneko, and H. Hirukawa, "Pushing Manipulation by Humanoid considering Two-Kinds of ZMPs," Proc. of 2003 IEEE International Conference on Robotics and Automation (ICRA'03), pp.1627-1632, 2003.

[5] T. Takubo, K. Inoue and T. Arai, "Pushing an Object Considering the Hand Reflect Forces by Humanoid Robot in Dynamic Walking," Proc. of IEEE Int. Conf. on Robotics and Automation, pp.1718-1723, 2005.

[6] C. Zhu, Y. Aiyama, T. Chawanya, and T. Arai, "Releasing Manipulation," Proc. of the IEEE/RSJ Int. Conf. on Intelligent Robots and Systems (IROS'96), pp.911-916, 1996.

[7] Takaaki Matsumoto, Atsushi Konno, Linlin Gou and Masaru Uchiyama, A Humanoid Robot that Breaks Wooden Boards Applying Impulsive Force, Proc. of the 2005 IEEE/RSJ International Conference on Intelligent Robots and Systems, pp. 5919 - 5924, 2006.

[8] Arisumi, H., Chardonnet, J-R., Kheddar, A., and Yokoi, K., "Dynamic Lifting Motion of Humanoid Robots," Proc. of the IEEE International Conference on Robotics and Automation, pp. 2661- 2667, 2007.

[9] Wen-Teng Cheng, "Synthesis of Universal Motion Curves in Generalized Model," Journal of Mechanical Design, Volume 124, Issue 2, pp. 284-293, 2002

[10] S. Nakaoka, S. Hattori, F. Kanehiro, S. Kajita and H. Hirukawa, "Constraint-based Dynamics Simulator for Humanoid Robots with Shock Absorbing Mechanisms," Proc. of the 2007 IEEE/RSJ International Conference on Intelligent Robots and Systems (IROS' 07), pp.3641-3647, 2007.

[11] T. Nakamura, H. Arisumi, and K. Yokoi, "Passing through a Door by a Humanoid robot - Door Modeling -," Proc. of the 9th SICE System Integration Division Annual Conference (SI2008), 3E3-1, 2008 (in Japanese). 